\title{
Constraining Non-Standard Dark Matter-Nucleon Interactions with IceCube
}

\section{The IceCube Collaboration}

(a complete list of authors can be found at the end of the proceedings)

E-mail: lilly.peters@icecube.wisc.edu

\begin{abstract}
After scattering off nuclei in the Sun, dark matter particles can be gravitationally captured by the Sun, accumulate in the Sun's core and annihilate into Standard Model particles. Neutrinos originating from these annihilations can be detected by the IceCube Neutrino Observatory, located at the South Pole. Due to the non-observation of these neutrinos, constraints on the standard spin-dependent and spin-independent dark matter-nucleon scattering cross sections have been placed. Based on these constraints, we present upper limits on the coupling constants of the non-relativistic effective theory of dark matter-nucleon interactions, including velocity and momentum dependent interactions.
\end{abstract}

Corresponding authors: Lilly Peters ${ }^{1,2 *}$, Koun $\mathrm{Choi}^{2}$, Mehr Un Nisa ${ }^{3}$

${ }^{1}$ III. Physikalisches Institut, RWTH Aachen University, D-52056 Aachen, Germany

${ }^{2}$ Dept. of Physics, Sungkyunkwan University, Korea

${ }^{3}$ Michigan State University

${ }^{*}$ Presenter

$37^{\text {th }}$ International Cosmic Ray Conference (ICRC 2021)

July 12th - 23rd, 2021

Online - Berlin, Germany 


\section{Introduction}

Although there is compelling evidence for the existence of dark matter (DM), its nature remains unknown. To explain observations, a variety of theories provide candidate particles [1]. There are three complementary ways to search for DM - collider searches, direct and indirect detection. Direct detection aims to determine interactions of DM particles with nuclei, typically with the goal of setting constraints on spin-dependent (SD) and spin-independent (SI) interaction terms. However, there also are approaches to cover the full set of operators in a non-relativistic effective theory, including momentum- and velocity-dependent operators [2]. In recent years, there have been several efforts from direct detection experiments like CRESST-II [3], Xenon100 [4], SuperCDMS [5] and DEAP-3600 [6] to constrain the coupling constants of the theory. With the IceCube Neutrino Observatory we are able to set limits via indirect detection based on DM capture in the Sun.

\section{Theory}

\subsection{Effective Theory of Dark Matter-Nucleon Interactions}

We will briefly summarize the effective theory of DM-nucleon interactions following [2]. The amplitude for elastic DM-nucleon scattering is restricted by several symmetries. In particular, Galilean invariance imposes that the scattering amplitude can only depend on combinations of the momentum transferred from the nucleon to the DM particle $\mathbf{q}$ and the relative incoming velocity $\mathbf{v}=\mathbf{v}_{\chi}-\mathbf{v}_{N}$ which is the incoming velocity of the DM particle $\chi$ in the rest frame of the nucleon $N$. In addition, the interactions must be hermitian, thus it is useful to work with hermitian quantities and to introduce $\mathbf{v}^{\perp}=\mathbf{v}+\mathbf{q} / 2 \mu_{N}$ with the reduced DM-nucleon mass $\mu_{N}$. All interaction operators can be written as a combination of $i \mathbf{q}, \mathbf{v}^{\perp}$, the DM $\operatorname{spin} \mathbf{S}_{\chi}$ and the nucleon $\operatorname{spin} \mathbf{S}_{N}$. They are listed in table 1 imposing that they are at most linear in $\mathbf{S}_{\chi}, \mathbf{S}_{N}$ and $\mathbf{v}^{\perp}$. It is assumed that the mediating particle has spin 0 or spin 1 and is heavy compared to the momentum transfer.

The interaction Langrangian has the form

$$
\mathcal{L}_{i n t}=\sum_{N=n, p} \sum_{i} c_{i}^{N} O_{i} \chi^{+} \chi^{-} N^{+} N^{-}
$$

where $\chi^{ \pm}, N^{ \pm}$are the fields involving only creation or annihilation fields and $c_{i}^{p}$ and $c_{i}^{n}$ denote the coupling constants for protons and neutrons and have mass dimension -2 [7]. Instead of proton and neutron couplings we will present the results in terms of isoscalar $c^{0}=c^{p}+c^{n}$ and isovector $c^{1}=c^{p}-c^{n}$ couplings.

Forming a Lagrangian from the operators in table 1 and performing a multipole expansion of the nuclear charges and currents lead to six nuclear response operators contributing to the transition probability, namely $M, \Sigma^{\prime}, \Sigma^{\prime \prime}, \Phi^{\prime \prime}, \tilde{\Phi}^{\prime}$ and $\Delta$.

Finally the differential cross-section for DM scattering off a nucleon of type $\mathrm{N}$ can be written as

$$
\begin{aligned}
\frac{d \sigma_{N}}{d E_{R}}\left(\omega^{2}, q^{2}\right)=\frac{2 m_{N}}{\omega^{2}} \frac{1}{2 J+1} & \sum_{\tau, \tau^{\prime}}\left[\sum_{k=M, \Sigma^{\prime}, \Sigma^{\prime \prime}} R_{k}^{\tau \tau^{\prime}}\left(v_{T}^{\perp 2}, \frac{q^{2}}{m_{N}^{2}}\right) W_{k}^{\tau \tau^{\prime}}\left(q^{2}\right)\right. \\
& \left.+\frac{q^{2}}{m_{N}^{2}} \sum_{k=\Phi^{\prime \prime}, \Phi^{\prime \prime} M, \tilde{\Phi}^{\prime}, \Delta, \Delta \Sigma^{\prime}} R_{k}^{\tau \tau^{\prime}}\left(v_{T}^{\perp 2}, \frac{q^{2}}{m_{N}^{2}}\right) W_{k}^{\tau \tau^{\prime}}\left(q^{2}\right)\right]
\end{aligned}
$$




\begin{tabular}{|c|c|}
\hline$O_{1}=\mathbb{1}_{\chi N}$ & $O_{11}=i \hat{\mathbf{S}}_{\chi} \cdot \frac{\hat{\mathbf{q}}}{m_{N}} \mathbb{1}_{\mathbb{N}}$ \\
\hline$O_{3}=i \hat{\mathbf{S}}_{N} \cdot\left(\frac{\hat{\mathbf{q}}}{m_{N}} \times \hat{\mathbf{v}}^{\perp}\right) \mathbb{1}_{\chi}$ & $O_{12}=\hat{\mathbf{S}}_{\chi} \cdot\left(\hat{\mathbf{S}}_{N} \times \hat{\mathbf{v}}^{\perp}\right)$ \\
\hline$O_{4}=\hat{\mathbf{S}}_{\chi} \cdot \hat{\mathbf{S}}_{N}$ & $O_{13}=i\left(\hat{\mathbf{S}}_{\chi} \cdot \hat{\mathbf{v}}^{\perp}\right)\left(\hat{\mathbf{S}}_{N} \cdot \frac{\hat{\mathbf{q}}}{m_{N}}\right)$ \\
\hline$O_{5}=i \hat{\mathbf{S}}_{\chi} \cdot\left(\frac{\hat{\mathbf{q}}}{m_{N}} \times \hat{\mathbf{v}}^{\perp}\right) \mathbb{1}_{\mathbb{N}}$ & $O_{14}=i\left(\hat{\mathbf{S}}_{\chi} \cdot \frac{\hat{\mathbf{q}}}{m_{N}}\right)^{\prime}\left(\hat{\mathbf{S}}_{N} \cdot \hat{\mathbf{v}}^{\perp}\right)$ \\
\hline$O_{6}=\left(\hat{\mathbf{S}}_{\chi} \cdot \frac{\hat{\mathbf{q}}}{m_{N}}\right)\left(\hat{\mathbf{S}}_{N} \cdot \frac{\hat{\mathbf{q}}}{m_{N}}\right)$ & $O_{15}=-\left(\hat{\mathbf{S}}_{\chi} \cdot \frac{\hat{\mathbf{q}}}{m_{N}}\right)\left[\left(\hat{\mathbf{S}}_{N} \times \hat{\mathbf{v}}^{\perp}\right) \cdot \frac{\hat{\mathbf{q}}}{m_{N}}\right]$ \\
\hline$O_{7}=\hat{\mathbf{S}}_{N} \cdot \hat{\mathbf{v}}^{\perp} \mathbb{1}_{\chi}$ & $O_{17}=i \frac{\hat{\mathbf{q}}}{m_{N}} \cdot \mathcal{S} \cdot \hat{\mathbf{v}}^{\perp} \mathbb{1}_{\mathbb{N}}$ \\
\hline$O_{8}=\hat{\mathbf{S}}_{\chi} \cdot \hat{\mathbf{v}}^{\perp} \mathbb{1}_{\mathbb{N}}$ & $O_{18}=i \frac{\hat{\mathbf{q}}}{m_{N}} \cdot \mathcal{S} \cdot \hat{\mathbf{S}}_{N}$ \\
\hline$O_{9}=i \hat{\mathbf{S}}_{\chi} \cdot\left(\hat{\mathbf{S}}_{N} \times \frac{\hat{\mathbf{q}}}{m_{N}}\right)$ & $O_{19}=\frac{\hat{\mathbf{q}}}{m_{N}} \cdot \mathcal{S} \cdot \frac{\hat{\mathbf{q}}}{m_{N}}$ \\
\hline$O_{10}=i \hat{\mathbf{S}}_{N} \cdot \frac{\hat{\mathbf{q}}}{m_{N}} \mathbb{1}_{\chi}$ & $O_{20}=\left(\hat{\mathbf{S}}_{N} \times \frac{\hat{\mathbf{q}}}{m_{N}}\right) \cdot \mathcal{S} \cdot \frac{\hat{\mathbf{q}}}{m_{N}}$ \\
\hline
\end{tabular}

Table 1: Non-relativistic quantum mechanical operators that are at most linear in $\mathbf{S}_{\chi}, \mathbf{S}_{N}$ and $\mathbf{v}^{\perp} . O_{2}$ is quadratic in $\mathbf{v}^{\perp}$ and $O_{16}$ a linear combination of $O_{12}$ and $O_{15}$, thus they are not considered here. $O_{1}$ and $\mathrm{O}_{4}$ correspond to the standard SI and SD interactions. By introducing the nucleon mass $m_{N}$ all operators have the same mass dimension. Operators 17 - 20 can only arise for spin $1 \mathrm{DM}$ and the symbol $\mathcal{S}$ denotes a symmetric combination of spin 1 polarisation vectors. In addition, $O_{19}$ and $O_{20}$ can only appear if the interaction is mediated by a vector mediator [8]

where the DM response functions $R_{k}^{\tau \tau^{\prime}}$ depend on the DM-nucleon interaction strength [7]. The isotope-dependent nuclear response functions $W_{k}^{\tau \tau^{\prime}}$ have been calculated for the 16 most abundant elements in the sun through numerical shell model calculations in [7].

\subsection{Dark Matter Capture in the Sun}

DM particles of the Milky Way DM halo can scatter off nuclei in the Sun and be gravitationally captured if they scatter from a velocity $\omega$ to a velocity smaller than the local escape velocity $v_{\odot}^{\text {esc }}(r)$.

The capture rate for nuclei of type $\mathrm{N}$ is given by

$$
\begin{gathered}
C_{\text {cap }}^{N}=n_{\chi} \int_{0}^{R_{\odot}} d r 4 \pi r^{2} n_{N}(r) \int_{0}^{\infty} d u 4 \pi u^{2} f_{\odot}(u) \frac{u^{2}+v_{\odot}^{\mathrm{esc}}(r)^{2}}{u} \int_{E_{\min }}^{E_{\max }} d E_{R} \frac{d \sigma_{N}}{d E_{R}} \theta(\Delta E) \\
E_{\min }=\frac{1}{2} M_{\chi} u^{2} \quad E_{\max }=\frac{2 \mu_{N}^{2}}{m_{N}}\left(u^{2}+v_{\odot}^{\mathrm{esc}}(r)^{2}\right) \quad \Delta E=E_{\max }-E_{\min }
\end{gathered}
$$

where $u$ is the velocity of the DM particle at $r \rightarrow \infty$ in the solar frame such that $\omega=\sqrt{u^{2}+v_{\odot}^{\text {esc }}(r)^{2}}$ [7]. We assume that the DM velocities follow a Maxwell-Boltzmann distribution in the galactic frame without a cut-off at the galactic escape velocity. We use $v_{\odot}=220 \mathrm{~km} / \mathrm{s}$ for the galactic orbital speed in the solar position, and $\sigma_{v}=270 \mathrm{~km} / \mathrm{s}$ for the velocity dispersion. To get the total capture rate $C_{\text {cap }}$, we sum equation (3) for each of the 16 most abundant elements in the Sun. The elemental abundances $n_{N}$ are taken from the B16 GS98 model [9]. Furthermore we assume the DM halo number density to be $n_{\chi}=0.3 \mathrm{GeV} / \mathrm{cm}^{3} / M_{\chi}$. For a discussion of the astrophysical uncertainties see section 4.2 . 


\section{Capture Rate Limits from IceCube Solar Dark Matter Analyses}

When DM particles are captured in the Sun, they can sink into the Sun's core and annihilate into Standard Model final states. Neglecting evaporation yields the following differential equation describing the number of DM particles in the Sun

$$
\frac{d N}{d t}=C_{\text {cap }}-C_{\text {ann }} N^{2}
$$

with the general solution $\Gamma_{A}=\frac{1}{2} C_{\mathrm{cap}} \tanh ^{2}\left(\sqrt{C_{\mathrm{cap}} C_{\mathrm{ann}}} t\right)$ [10]. Neutrinos originating from these annihilations can potentially be observed by neutrino telescopes like the IceCube Neutrino Observatory. IceCube is a cubic-kilometer sized neutrino detector installed in the ice at the geographic South Pole between depths of $1450 \mathrm{~m}$ and $2450 \mathrm{~m}$, completed in 2010 [11]. Neutrino reconstruction relies on the optical detection of Cherenkov radiation emitted by charged particles produced in the interactions of neutrinos in the surrounding ice or the nearby bedrock.

IceCube searches for an excess of neutrino events correlated with the direction of the Sun in order to detect signatures of Solar DM annihilation. The results published based on three years of data were able to constrain DM-nucelon scattering for three annihilation channels $\left(\tau^{+} \tau^{-}, W^{+} W^{-}\right.$, $b \bar{b}$ ) for $\mathrm{DM}$ masses up to $10 \mathrm{TeV}$ [12]. Recently, utilizing IceCube DeepCore data and new cuts, an updated analysis using seven years of data pushes IceCube sensitivity at the lower energy end of the DM mass range down to $5 \mathrm{GeV}$ (publication under preparation), and also constrains DM annihilating directly to neutrinos.

\section{Results}

\subsection{Capture rates}

To calculate the capture rate for each operator, the value of the coupling constant was set to $c_{i}=10^{-3} m_{v}^{2}$ with $m_{v}=246.2 \mathrm{GeV}$, while all other coupling constants were set to zero. The value is arbitrary as the capture rate is proportional to the coupling constant squared and the ratio of the two will be used as a conversionfactor in section 4.3. There are six isotopes that are responsible for the leading contribution for at least one interaction operator in a certain DM mass range, namely ${ }^{1} \mathrm{H},{ }^{4} \mathrm{He},{ }^{14} \mathrm{~N},{ }^{16} \mathrm{O},{ }^{27} \mathrm{Al}$ and ${ }^{56} \mathrm{Fe}$. The most important isotope for each operator is determined by a compromise between the elemental abundance, the properties of the nuclear response operators and kinematic factors that depend on the DM and the nucleon mass.

\subsection{Systematic uncertainties}

The most prominent uncertainty in terms of astrophysical uncertainties is the local DM density that we assume to be $0.3 \mathrm{GeV} / \mathrm{cm}^{3}$, a widely-used value [13]. Recent measurements have best-fit values in the range $0.2-0.6 \mathrm{GeV} / \mathrm{cm}^{3}[13]$. The capture rates and the limits in the next section can be adapted to a different density by scaling with the ratio of the two numbers.

The capture process is sensitive to deviations from the assumed Maxwellian velocity distribution. Figure 1 shows the change in capture rate for a range of $v_{\odot}$ from 200 to $280 \mathrm{~km} / \mathrm{s}$, while $\sigma_{v}$ is set to $\sqrt{3 / 2} v_{\odot}$. Since Operator 7 is dominated by hydrogen, it is sensitive to changes in the velocity distribution earlier (at smaller DM masses) than operator 15, which is dominated by iron. 

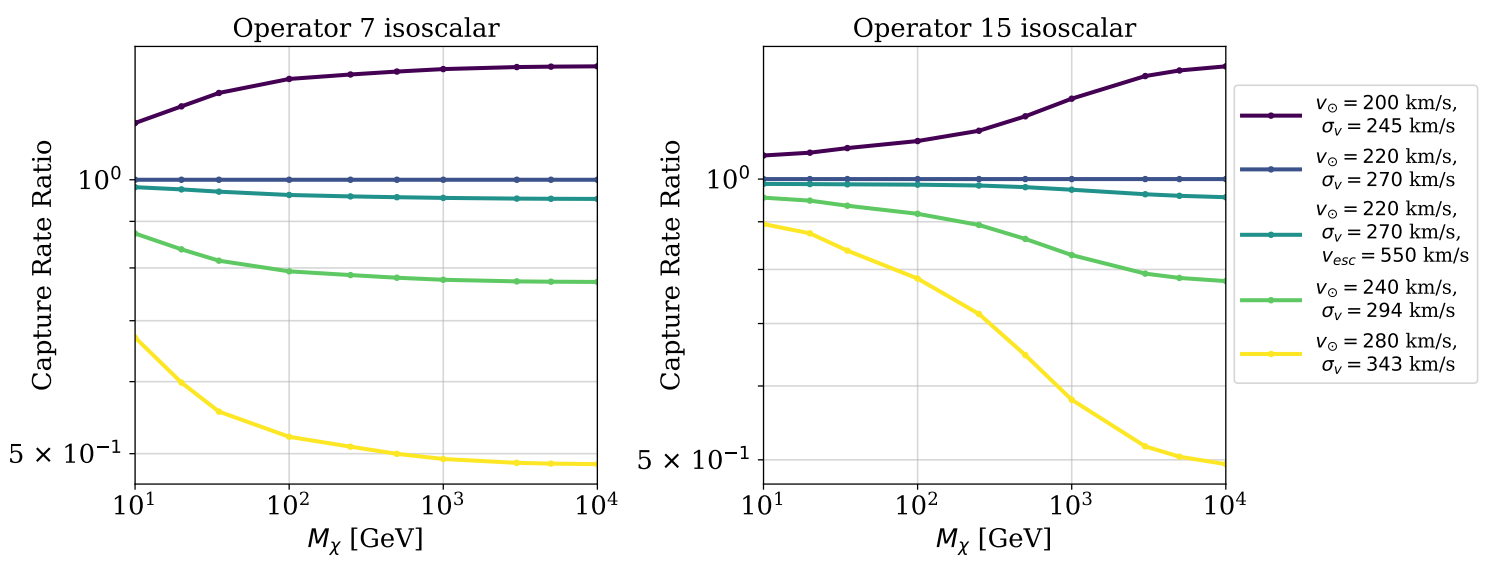

Figure 1: Capture rate ratio to the standard assumption as a function of the DM mass for different $f_{\odot}(u)$ with assumed $v_{\odot}, \sigma_{v}$ as specified in the legend. The value of $\sigma_{v}$ is set to $\sqrt{3 / 2} v_{\odot}$. If no value for the galactic escape velocity is specified, the cut-off has been neglected. Exemplary, operator 7 and 15 are shown, dominated by hydrogen and iron, respectively.

Another uncertainty is the elemental composition of the sun. Especially the abundance of the respective dominant isotope is important. For a discussion of the uncertainties in the solar model see [9].

IceCube sensitivity to DM annihilation is also impacted by various detector systematics, which are included in the annihilation rate limits and propagate into the upper limits on the coupling constants.

\subsection{Limits on the Coupling Constants}

We can now use the computed capture rates to convert the capture rate limits from the IceCube analyses described in section 3 into limits on the coupling constants. The results are shown in figure 2 and 3 for isoscalar and isovector interactions. For simplicity we only present results for DM particles with spin $j_{\mathcal{X}}=\frac{1}{2}$. The limits are compared to the direct detection experiments CRESST-II [3], Xenon100 [4], DEAP-3600 [6], CDMS II and SuperCDMS [5]. All exclusion limits are at 90 $\%$ confidence level. CRESST-II is not competitive in the considered mass range but provides the strongest constraints below $5 \mathrm{GeV}$ and is thus shown for completeness. A comparison to LUX [14] is not feasible since they present their results in terms of proton/neutron instead of isoscalar/isovector couplings. Because direct detection experiments are restricted to the isotopes in their detectors, they cannot always set constraints on all interaction types. We observe that whether the strongest constraint comes from direct detection or IceCube is highly dependent on the operator. Notably, for Operator 4, 7 and 14 IceCube sets the most stringent limits for a large mass range, for the first two even with the soft channel analysis.

\section{Conclusion and Outlook}

We have reported upper limits on the isocalar and isovector coupling constants of the nonrelativistic effective theory of DM-nucleon interactions for DM particles with spin $1 / 2$. We want to 

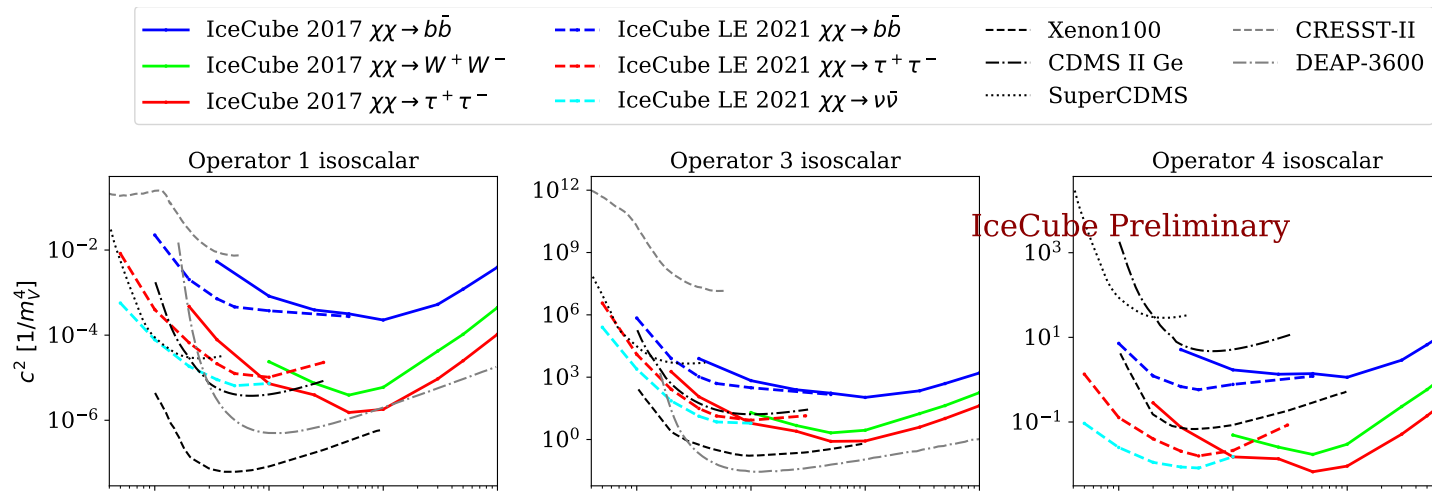

Operator 4 isoscalar
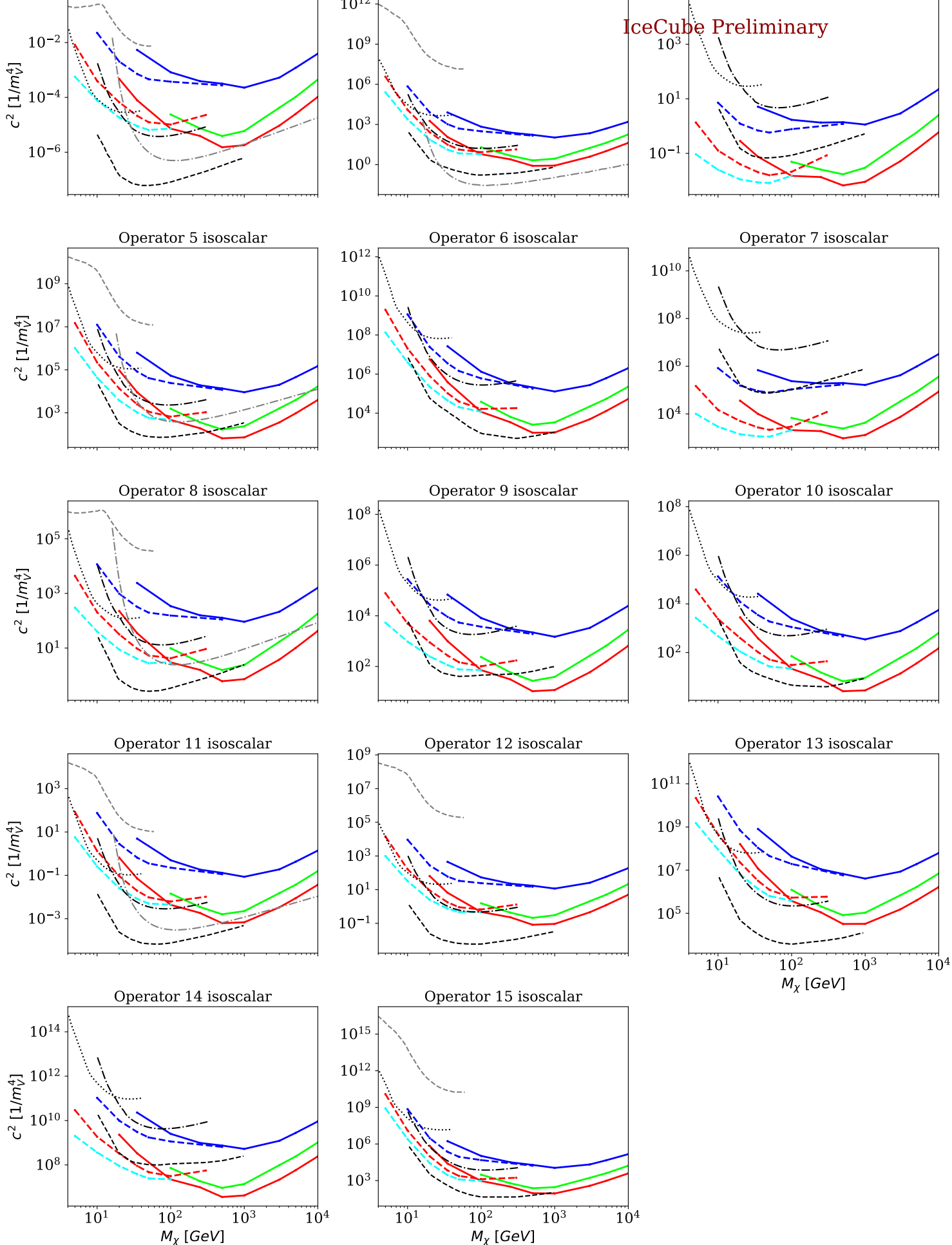

Figure 2: Exclusion limits on the isoscalar coupling constants at $90 \%$ confidence level. 


\begin{tabular}{|c|c|c|c|}
\hline - IceCube $2017 \chi \chi \rightarrow b \bar{b}$ & - IceCube $2017 \chi \chi \rightarrow \tau^{+} \tau^{-}$ & --- IceCube LE $2021 \chi \chi \rightarrow \tau^{+} \tau^{-}$ & -... DEAP-3600 \\
\hline IceCube $2017 \chi \chi \rightarrow W^{+} W^{-}$ & --- IceCube LE $2021 \chi \chi \rightarrow b \bar{b}$ & $---\quad$ IceCube LE $2021 \chi \chi \rightarrow \nu \bar{\nu}$ & \\
\hline
\end{tabular}
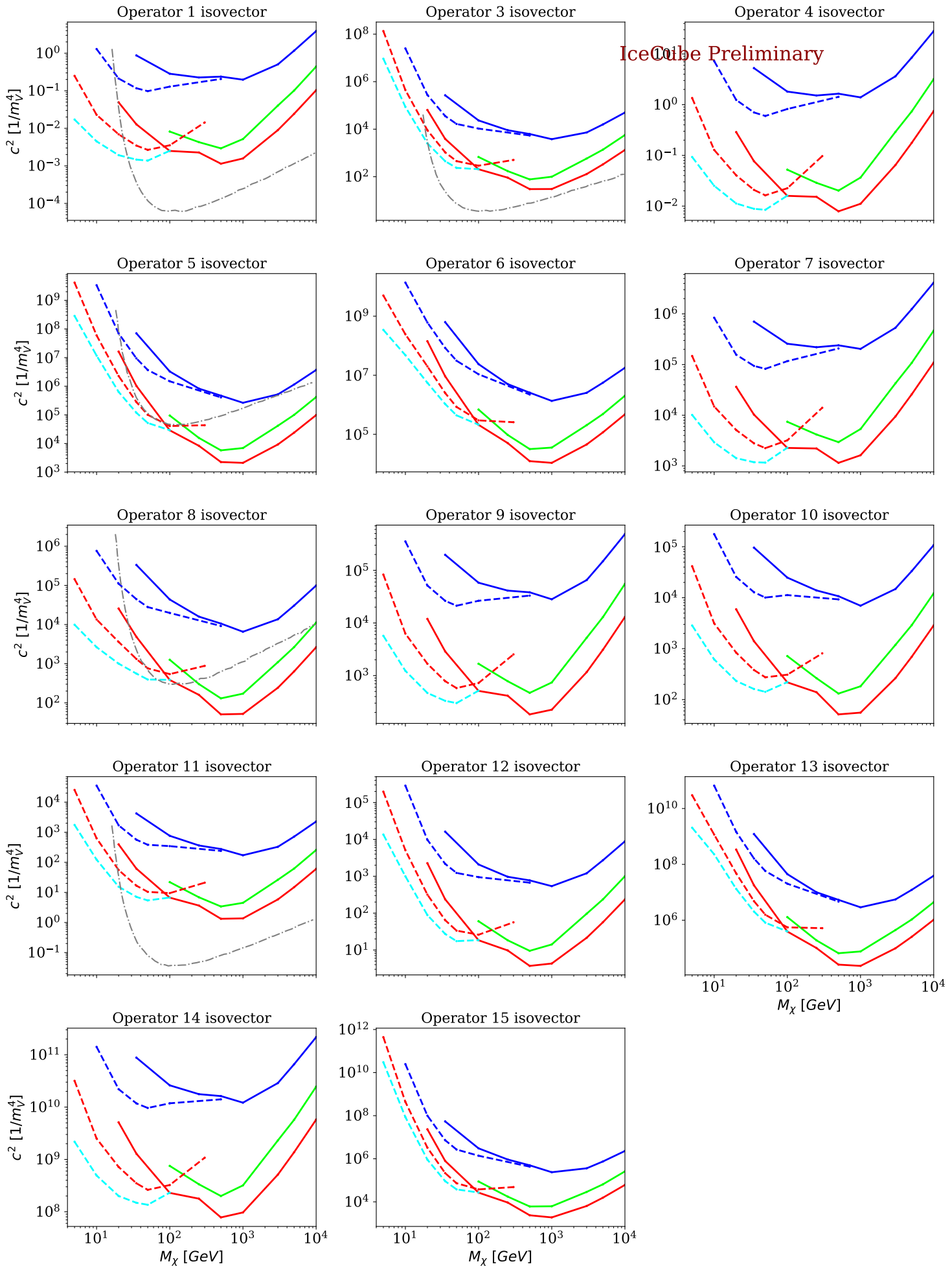

Figure 3: Exclusion limits on the isovector coupling constants at $90 \%$ confidence level. 
stress that the theoretical framework presented in section 2.1 is also valid for other spins and that the analysis can easily be extended.

The shown results are based on annihilation rate limits from two previous IceCube analyses, one from 2017 using three years of data [12] and the other from 2021 focussing on the low energy regime (publication under preparation). Including new analyses like [15] could further improve the limits presented here.

\section{References}

[1] G. Bertone, D. Hooper, and J. Silk Phys. Rept. 405 (2005) 279-390.

[2] A. L. Fitzpatrick, W. Haxton, E. Katz, N. Lubbers, and Y. Xu JCAP 02 (2013) 004.

[3] CRESST Collaboration, G. Angloher et al. Eur. Phys. J. C 79 no. 1, (2019) 43.

[4] XENON Collaboration, E. Aprile et al. Phys. Rev. D 96 no. 4, (2017) 042004.

[5] SuperCDMS Collaboration, K. Schneck et al. Phys. Rev. D 91 no. 9, (2015) 092004.

[6] DEAP Collaboration, P. Adhikari et al. Phys. Rev. D 102 no. 8, (2020) 082001.

[7] R. Catena and B. Schwabe JCAP 04 (2015) 042.

[8] R. Catena, K. Fridell, and M. B. Krauss JHEP 08 (2019) 030.

[9] N. Vinyoles, A. M. Serenelli, F. L. Villante, S. Basu, J. Bergström, M. C. Gonzalez-Garcia, M. Maltoni, C. Peña Garay, and N. Song Astrophys. J. 835 no. 2, (2017) 202.

[10] G. Jungman, M. Kamionkowski, and K. Griest Phys. Rept. 267 (1996) 195-373.

[11] IceCube Collaboration, M. G. Aartsen et al. JINST 12 no. 03, (2017) P03012.

[12] IceCube Collaboration, M. G. Aartsen et al. Eur. Phys. J. C 77 no. 3, (2017) 146. [Erratum: Eur.Phys.J.C 79, 214 (2019)].

[13] E. Del Nobile arXiv e-prints (Apr., 2021) arXiv:2104.12785.

[14] LUX Collaboration, D. S. Akerib et al. arXiv e-prints (Feb., 2021) arXiv:2102.06998.

[15] IceCube Collaboration PoS ICRC2021 (these proceedings) 541. 


\section{Full Author List: IceCube Collaboration}

R. Abbasi ${ }^{17}$, M. Ackermann ${ }^{59}$, J. Adams ${ }^{18}$, J. A. Aguilar ${ }^{12}$, M. Ahlers ${ }^{22}$, M. Ahrens ${ }^{50}$, C. Alispach ${ }^{28}$, A. A. Alves Jr. ${ }^{31}$, N. M. $\mathrm{Amin}^{42}$, R. An ${ }^{14}$, K. Andeen ${ }^{40}$, T. Anderson ${ }^{56}$, G. Anton ${ }^{26}$, C. Argüelles ${ }^{14}$, Y. Ashida ${ }^{38}$, S. Axani ${ }^{15}$, X. Bai ${ }^{46}$, A. Balagopal V. ${ }^{38}$, A. Barbano ${ }^{28}$, S. W. Barwick ${ }^{30}$, B. Bastian ${ }^{59}$, V. Basu ${ }^{38}$, S. Baur ${ }^{12}$, R. Bay ${ }^{8}$, J. J. Beatty ${ }^{20,21}$, K.-H. Becker ${ }^{58}$, J. Becker Tjus ${ }^{11}$, C. Bellenghi $^{27}$, S. BenZvi ${ }^{48}$, D. Berley ${ }^{19}$, E. Bernardini ${ }^{59}, 60$, D. Z. Besson ${ }^{34,61}$, G. Binder ${ }^{8,9}$, D. Bindig ${ }^{58}$, E. Blaufuss ${ }^{19}$, S. Blot ${ }^{59}$, M. Boddenberg ${ }^{1}$, F. Bontempo ${ }^{31}$, J. Borowka ${ }^{1}$, S. Böser ${ }^{39}$, O. Botner ${ }^{57}$, J. Böttcher ${ }^{1}$, E. Bourbeau ${ }^{22}$, F. Bradascio ${ }^{59}$, J. Braun ${ }^{38}$, S. Bron $^{28}$, J. Brostean-Kaiser ${ }^{59}$, S. Browne ${ }^{32}$, A. Burgman ${ }^{57}$, R. T. Burley ${ }^{2}$, R. S. Busse ${ }^{41}$, M. A. Campana ${ }^{45}$, E. G. Carnie-Bronca ${ }^{2}$, C. Chen ${ }^{6}$, D. Chirkin ${ }^{38}$, K. Choi ${ }^{52}$, B. A. Clark ${ }^{24}$, K. Clark ${ }^{33}$, L. Classen ${ }^{41}$, A. Coleman ${ }^{42}$, G. H. Collin ${ }^{15}$, J. M. Conrad ${ }^{15}$, P. Coppin $^{13}$, P. Correa ${ }^{13}$, D. F. Cowen ${ }^{55}, 56$, R. Cross ${ }^{48}$, C. Dappen ${ }^{1}$, P. Dave ${ }^{6}$, C. De Clercq ${ }^{13}$, J. J. DeLaunay ${ }^{56}$, H. Dembinski ${ }^{42}$, K. Deoskar $^{50}$, S. De Ridder ${ }^{29}$, A. Desai ${ }^{38}$, P. Desiati ${ }^{38}$, K. D. de Vries ${ }^{13}$, G. de Wasseige ${ }^{13}$, M. de With ${ }^{10}$, T. DeYoung ${ }^{24}$, S. Dharani $^{1}$, A. Diaz ${ }^{15}$, J. C. Díaz-Vélez ${ }^{38}$, M. Dittmer ${ }^{41}$, H. Dujmovic ${ }^{31}$, M. Dunkman ${ }^{56}$, M. A. DuVernois ${ }^{38}$, E. Dvorak ${ }^{46}$, T. Ehrhardt ${ }^{39}$, P. Eller $^{27}$, R. Engel $^{31,32}$, H. Erpenbeck ${ }^{1}$, J. Evans ${ }^{19}$, P. A. Evenson ${ }^{42}$, K. L. Fan ${ }^{19}$, A. R. Fazely ${ }^{7}$, S. Fiedlschuster ${ }^{26}$, A. T. Fienberg ${ }^{56}$, K. Filimonov ${ }^{8}$, C. Finley ${ }^{50}$, L. Fischer ${ }^{59}$, D. Fox ${ }^{55}$, A. Franckowiak ${ }^{11,59}$, E. Friedman ${ }^{19}$, A. Fritz ${ }^{39}$, P. Fürst ${ }^{1}$, T. K. Gaisser ${ }^{42}$, J. $^{3}$ Gallagher $^{37}$, E. Ganster ${ }^{1}$, A. Garcia ${ }^{14}$, S. Garrappa ${ }^{59}$, L. Gerhardt ${ }^{9}$, A. Ghadimi ${ }^{54}$, C. Glaser ${ }^{57}$, T. Glauch ${ }^{27}$, T. Glüsenkamp ${ }^{26}$, A. Goldschmidt $^{9}$, J. G. Gonzalez ${ }^{42}$, S. Goswami ${ }^{54}$, D. Grant ${ }^{24}$, T. Grégoire ${ }^{56}$, S. Griswold ${ }^{48}$, M. Gündüz ${ }^{11}$, C. Günther ${ }^{1}$, C. Haack ${ }^{27}$,

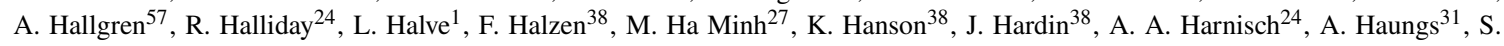
Hauser $^{1}$, D. Hebecker ${ }^{10}$, K. Helbing ${ }^{58}$, F. Henningsen ${ }^{27}$, E. C. Hettinger ${ }^{24}$, S. Hickford ${ }^{58}$, J. Hignight ${ }^{25}$, C. Hill ${ }^{16}$, G. C. Hill ${ }^{2}$, K. D.

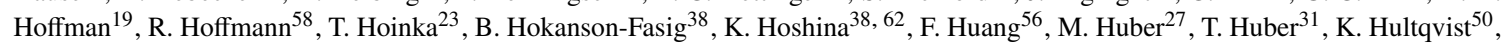
M. Hünnefeld ${ }^{23}$, R. Hussain ${ }^{38}$, S. In ${ }^{52}$, N. Iovine ${ }^{12}$, A. Ishihara ${ }^{16}$, M. Jansson ${ }^{50}$, G. S. Japaridze ${ }^{5}$, M. Jeong ${ }^{52}$, B. J. P. Jones ${ }^{4}$, D. Kang ${ }^{31}$, W. Kang ${ }^{52}$, X. Kang ${ }^{45}$, A. Kappes ${ }^{41}$, D. Kappesser ${ }^{39}$, T. Karg ${ }^{59}$, M. Karl ${ }^{27}$, A. Karle ${ }^{38}$, U. Katz ${ }^{26}$, M. Kauer ${ }^{38}$, M. Kellermann ${ }^{1}$, J. L. Kelley $^{38}$, A. Kheirandish ${ }^{56}$, K. Kin ${ }^{16}$, T. Kintscher ${ }^{59}$, J. Kiryluk ${ }^{51}$, S. R. Klein ${ }^{8,9}$, R. Koirala ${ }^{42}$, H. Kolanoski ${ }^{10}$, T. Kontrimas ${ }^{27}$, L. Köpke $^{39}$, C. Kopper ${ }^{24}$, S. Kopper ${ }^{54}$, D. J. Koskinen ${ }^{22}$, P. Koundal ${ }^{31}$, M. Kovacevich ${ }^{45}$, M. Kowalski ${ }^{10,59}$, T. Kozynets ${ }^{22}$, E. Kun ${ }^{11}$, N. Kurahashi ${ }^{45}$, N. Lad ${ }^{59}$, C. Lagunas Gualda ${ }^{59}$, J. L. Lanfranchi ${ }^{56}$, M. J. Larson ${ }^{19}$, F. Lauber ${ }^{58}$, J. P. Lazar ${ }^{14,38}$, J. W. Lee ${ }^{52}$, K. Leonard $^{38}$, A. Leszczyńska ${ }^{32}$, Y. Li ${ }^{56}$, M. Lincetto ${ }^{11}$, Q. R. Liu ${ }^{38}$, M. Liubarska ${ }^{25}$, E. Lohfink ${ }^{39}$, C. J. Lozano Mariscal ${ }^{41}$, L. Lu ${ }^{38}$,

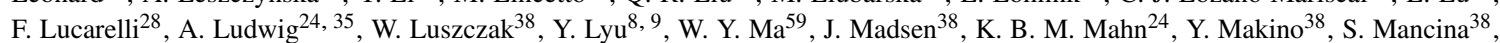

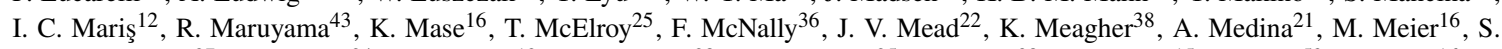
Meighen-Berger $^{27}$, J. Micallef ${ }^{24}$, D. Mockler ${ }^{12}$, T. Montaruli ${ }^{28}$, R. W. Moore ${ }^{25}$, R. Morse ${ }^{38}$, M. Moulai $^{15}$, R. Naab ${ }^{59}$, R. Nagai ${ }^{16}$, U. Naumann $^{58}$, J. Necker ${ }^{59}$, L. V. Nguyễn ${ }^{24}$, H. Niederhausen ${ }^{27}$, M. U. Nisa ${ }^{24}$, S. C. Nowicki ${ }^{24}$, D. R. Nygren ${ }^{9}$, A. Obertacke Pollmann ${ }^{58}$, M. Oehler ${ }^{31}$, A. Olivas ${ }^{19}$, E. O'Sullivan ${ }^{57}$, H. Pandya ${ }^{42}$, D. V. Pankova ${ }^{56}$, N. Park ${ }^{33}$, G. K. Parker ${ }^{4}$, E. N. Paudel ${ }^{42}$, L. Paul ${ }^{40}$, C. Pérez de los $\operatorname{Heros}^{57}$, L. Peters ${ }^{1}$, J. Peterson ${ }^{38}$, S. Philippen ${ }^{1}$, D. Pieloth ${ }^{23}$, S. Pieper ${ }^{58}$, M. Pittermann ${ }^{32}$, A. Pizzuto ${ }^{38}$, M. Plum ${ }^{40}$, Y. Popovych $^{39}$, A. Porcelli ${ }^{29}$, M. Prado Rodriguez ${ }^{38}$, P. B. Price ${ }^{8}$, B. Pries ${ }^{24}$, G. T. Przybylski ${ }^{9}$, C. Raab ${ }^{12}$, A. Raissi ${ }^{18}$, M. Rameez $^{22}$, K. Rawlins $^{3}$, I. C. Rea ${ }^{27}$, A. Rehman ${ }^{42}$, P. Reichherzer ${ }^{11}$, R. Reimann ${ }^{1}$, G. Renzi ${ }^{12}$, E. Resconi ${ }^{27}$, S. Reusch ${ }^{59}$, W. Rhode ${ }^{23}$, M. Richman ${ }^{45}$, B. Riedel ${ }^{38}$, E. J. Roberts ${ }^{2}$, S. Robertson ${ }^{8,9}$, G. Roellinghoff ${ }^{52}$, M. Rongen ${ }^{39}$, C. Rott ${ }^{49}$, 52, T. Ruhe ${ }^{23}$, D. Ryckbosch ${ }^{29}$, D. Rysewyk $\mathrm{Cantu}^{24}$, I. Safa ${ }^{14,38}$, J. Saffer ${ }^{32}$, S. E. Sanchez Herrera ${ }^{24}$, A. Sandrock ${ }^{23}$, J. Sandroos ${ }^{39}$, M. Santander ${ }^{54}$, S. Sarkar ${ }^{44}$, S. Sarkar ${ }^{25}$, K. Satalecka $^{59}$, M. Scharf ${ }^{1}$, M. Schaufel ${ }^{1}$, H. Schieler ${ }^{31}$, S. Schindler ${ }^{26}$, P. Schlunder ${ }^{23}$, T. Schmidt ${ }^{19}$, A. Schneider ${ }^{38}$, J. Schneider ${ }^{26}$, F. G. Schröder ${ }^{31,42}$, L. Schumacher ${ }^{27}$, G. Schwefer ${ }^{1}$, S. Sclafani ${ }^{45}$, D. Seckel ${ }^{42}$, S. Seunarine ${ }^{47}$, A. Sharma ${ }^{57}$, S. Shefali ${ }^{32}$, M. Silva $^{38}$, B. Skrzypek ${ }^{14}$, B. Smithers ${ }^{4}$, R. Snihur ${ }^{38}$, J. Soedingrekso ${ }^{23}$, D. $\operatorname{Soldin}^{42}$, C. Spannfellner ${ }^{27}$, G. M. Spiczak ${ }^{47}$, C. Spiering ${ }^{59,61}$, J. Stachurska $^{59}$, M. Stamatikos ${ }^{21}$, T. Stanev ${ }^{42}$, R. Stein ${ }^{59}$, J. Stettner ${ }^{1}$, A. Steuer ${ }^{39}$, T. Stezelberger ${ }^{9}$, T. Stürwald ${ }^{58}$, T. Stuttard ${ }^{22}$, G. W. Sullivan $^{19}$, I. Taboada ${ }^{6}$, F. Tenholt ${ }^{11}$, S. Ter-Antonyan ${ }^{7}$, S. Tilav ${ }^{42}$, F. Tischbein ${ }^{1}$, K. Tollefson $^{24}$, L. Tomankova ${ }^{11}$, C. Tönnis ${ }^{53}$, S.

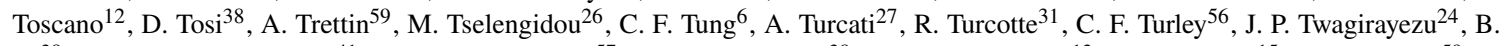
$\mathrm{Ty}^{38}$, M. A. Unland Elorrieta ${ }^{41}$, N. Valtonen-Mattila ${ }^{57}$, J. Vandenbroucke ${ }^{38}$, N. van Eijndhoven ${ }^{13}$, D. Vannerom ${ }^{15}$, J. van Santen ${ }^{59}, \mathrm{~S}$.

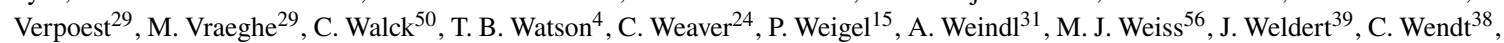
J. Werthebach ${ }^{23}$, M. Weyrauch ${ }^{32}$, N. Whitehorn ${ }^{24,35}$, C. H. Wiebusch ${ }^{1}$, D. R. Williams ${ }^{54}$, M. Wolf ${ }^{27}$, K. Woschnagg $^{8}$, G. Wrede ${ }^{26}$, J. Wulff ${ }^{11}$, X. W. Xu $u^{7}$, Y. Xu ${ }^{51}$, J. P. Yanez ${ }^{25}$, S. Yoshida ${ }^{16}$, S. Yu ${ }^{24}$, T. Yuan ${ }^{38}$, Z. Zhang ${ }^{51}$

${ }^{1}$ III. Physikalisches Institut, RWTH Aachen University, D-52056 Aachen, Germany

2 Department of Physics, University of Adelaide, Adelaide, 5005, Australia

${ }^{3}$ Dept. of Physics and Astronomy, University of Alaska Anchorage, 3211 Providence Dr., Anchorage, AK 99508, USA

${ }^{4}$ Dept. of Physics, University of Texas at Arlington, 502 Yates St., Science Hall Rm 108, Box 19059, Arlington, TX 76019, USA

5 CTSPS, Clark-Atlanta University, Atlanta, GA 30314, USA

${ }^{6}$ School of Physics and Center for Relativistic Astrophysics, Georgia Institute of Technology, Atlanta, GA 30332, USA

${ }^{7}$ Dept. of Physics, Southern University, Baton Rouge, LA 70813, USA

${ }^{8}$ Dept. of Physics, University of California, Berkeley, CA 94720, USA

${ }^{9}$ Lawrence Berkeley National Laboratory, Berkeley, CA 94720, USA

${ }^{10}$ Institut für Physik, Humboldt-Universität zu Berlin, D-12489 Berlin, Germany

${ }^{11}$ Fakultät für Physik \& Astronomie, Ruhr-Universität Bochum, D-44780 Bochum, Germany

12 Université Libre de Bruxelles, Science Faculty CP230, B-1050 Brussels, Belgium

13 Vrije Universiteit Brussel (VUB), Dienst ELEM, B-1050 Brussels, Belgium

14 Department of Physics and Laboratory for Particle Physics and Cosmology, Harvard University, Cambridge, MA 02138, USA

15 Dept. of Physics, Massachusetts Institute of Technology, Cambridge, MA 02139, USA 
${ }^{16}$ Dept. of Physics and Institute for Global Prominent Research, Chiba University, Chiba 263-8522, Japan

${ }^{17}$ Department of Physics, Loyola University Chicago, Chicago, IL 60660, USA

${ }^{18}$ Dept. of Physics and Astronomy, University of Canterbury, Private Bag 4800, Christchurch, New Zealand

${ }^{19}$ Dept. of Physics, University of Maryland, College Park, MD 20742, USA

${ }^{20}$ Dept. of Astronomy, Ohio State University, Columbus, OH 43210, USA

${ }^{21}$ Dept. of Physics and Center for Cosmology and Astro-Particle Physics, Ohio State University, Columbus, OH 43210, USA

${ }^{22}$ Niels Bohr Institute, University of Copenhagen, DK-2100 Copenhagen, Denmark

${ }^{23}$ Dept. of Physics, TU Dortmund University, D-44221 Dortmund, Germany

${ }^{24}$ Dept. of Physics and Astronomy, Michigan State University, East Lansing, MI 48824, USA

${ }^{25}$ Dept. of Physics, University of Alberta, Edmonton, Alberta, Canada T6G 2E1

${ }^{26}$ Erlangen Centre for Astroparticle Physics, Friedrich-Alexander-Universität Erlangen-Nürnberg, D-91058 Erlangen, Germany

${ }^{27}$ Physik-department, Technische Universität München, D-85748 Garching, Germany

${ }^{28}$ Département de physique nucléaire et corpusculaire, Université de Genève, CH-1211 Genève, Switzerland

${ }^{29}$ Dept. of Physics and Astronomy, University of Gent, B-9000 Gent, Belgium

${ }^{30}$ Dept. of Physics and Astronomy, University of California, Irvine, CA 92697, USA

${ }^{31}$ Karlsruhe Institute of Technology, Institute for Astroparticle Physics, D-76021 Karlsruhe, Germany

${ }^{32}$ Karlsruhe Institute of Technology, Institute of Experimental Particle Physics, D-76021 Karlsruhe, Germany

${ }^{33}$ Dept. of Physics, Engineering Physics, and Astronomy, Queen's University, Kingston, ON K7L 3N6, Canada

${ }^{34}$ Dept. of Physics and Astronomy, University of Kansas, Lawrence, KS 66045, USA

${ }^{35}$ Department of Physics and Astronomy, UCLA, Los Angeles, CA 90095, USA

${ }^{36}$ Department of Physics, Mercer University, Macon, GA 31207-0001, USA

${ }^{37}$ Dept. of Astronomy, University of Wisconsin-Madison, Madison, WI 53706, USA

${ }^{38}$ Dept. of Physics and Wisconsin IceCube Particle Astrophysics Center, University of Wisconsin-Madison, Madison, WI 53706, USA

${ }^{39}$ Institute of Physics, University of Mainz, Staudinger Weg 7, D-55099 Mainz, Germany

${ }^{40}$ Department of Physics, Marquette University, Milwaukee, WI, 53201, USA

${ }^{41}$ Institut für Kernphysik, Westfälische Wilhelms-Universität Münster, D-48149 Münster, Germany

42 Bartol Research Institute and Dept. of Physics and Astronomy, University of Delaware, Newark, DE 19716, USA

${ }^{43}$ Dept. of Physics, Yale University, New Haven, CT 06520, USA

${ }^{44}$ Dept. of Physics, University of Oxford, Parks Road, Oxford OX1 3PU, UK

${ }^{45}$ Dept. of Physics, Drexel University, 3141 Chestnut Street, Philadelphia, PA 19104, USA

${ }^{46}$ Physics Department, South Dakota School of Mines and Technology, Rapid City, SD 57701, USA

${ }^{47}$ Dept. of Physics, University of Wisconsin, River Falls, WI 54022, USA

${ }^{48}$ Dept. of Physics and Astronomy, University of Rochester, Rochester, NY 14627, USA

${ }^{49}$ Department of Physics and Astronomy, University of Utah, Salt Lake City, UT 84112, USA

50 Oskar Klein Centre and Dept. of Physics, Stockholm University, SE-10691 Stockholm, Sweden

${ }^{51}$ Dept. of Physics and Astronomy, Stony Brook University, Stony Brook, NY 11794-3800, USA

52 Dept. of Physics, Sungkyunkwan University, Suwon 16419, Korea

${ }^{53}$ Institute of Basic Science, Sungkyunkwan University, Suwon 16419, Korea

${ }^{54}$ Dept. of Physics and Astronomy, University of Alabama, Tuscaloosa, AL 35487, USA

${ }^{55}$ Dept. of Astronomy and Astrophysics, Pennsylvania State University, University Park, PA 16802, USA

56 Dept. of Physics, Pennsylvania State University, University Park, PA 16802, USA

${ }^{57}$ Dept. of Physics and Astronomy, Uppsala University, Box 516, S-75120 Uppsala, Sweden

${ }^{58}$ Dept. of Physics, University of Wuppertal, D-42119 Wuppertal, Germany

${ }^{59}$ DESY, D-15738 Zeuthen, Germany

${ }^{60}$ Università di Padova, I-35131 Padova, Italy

${ }^{61}$ National Research Nuclear University, Moscow Engineering Physics Institute (MEPhI), Moscow 115409, Russia

${ }^{62}$ Earthquake Research Institute, University of Tokyo, Bunkyo, Tokyo 113-0032, Japan

\section{Acknowledgements}

USA - U.S. National Science Foundation-Office of Polar Programs, U.S. National Science Foundation-Physics Division, U.S. National Science Foundation-EPSCoR, Wisconsin Alumni Research Foundation, Center for High Throughput Computing (CHTC) at the University of Wisconsin-Madison, Open Science Grid (OSG), Extreme Science and Engineering Discovery Environment (XSEDE), Frontera computing project at the Texas Advanced Computing Center, U.S. Department of Energy-National Energy Research Scientific Computing Center, Particle astrophysics research computing center at the University of Maryland, Institute for Cyber-Enabled Research at Michigan State University, and Astroparticle physics computational facility at Marquette University; Belgium - Funds for Scientific Research (FRS-FNRS and FWO), FWO Odysseus and Big Science programmes, and Belgian Federal Science Policy Office (Belspo); Germany - Bundesministerium für Bildung und Forschung (BMBF), Deutsche Forschungsgemeinschaft (DFG), Helmholtz Alliance for Astroparticle Physics (HAP), Initiative and Networking Fund of the Helmholtz Association, Deutsches Elektronen Synchrotron (DESY), and High Performance Computing cluster of the RWTH Aachen; Sweden - Swedish Research Council, Swedish Polar Research Secretariat, Swedish National Infrastructure for Computing (SNIC), and Knut and Alice Wallenberg Foundation; Australia - Australian 
Research Council; Canada - Natural Sciences and Engineering Research Council of Canada, Calcul Québec, Compute Ontario, Canada Foundation for Innovation, WestGrid, and Compute Canada; Denmark - Villum Fonden and Carlsberg Foundation; New Zealand Marsden Fund; Japan - Japan Society for Promotion of Science (JSPS) and Institute for Global Prominent Research (IGPR) of Chiba University; Korea - National Research Foundation of Korea (NRF); Switzerland - Swiss National Science Foundation (SNSF); United Kingdom - Department of Physics, University of Oxford. 